\title{
Vivência acadêmica: expectativas e desafios dos ingressantes e concluintes da graduação em enfermagem
}

\author{
Academic experience: expectations and challenges of freshmen and undergraduate \\ nursing graduates
}
Experiencia académica: expectativas y desafíos de estudiantes de primer año y graduados en enfermería

Tainara Lima Viana ${ }^{1 *}$, Adielly de Lima Souza ${ }^{1}$, Ivana Annely Cortez da Fonseca ${ }^{1}$.

\begin{abstract}
RESUMO
Objetivo: Compreender os sentimentos vivenciados pelos acadêmicos mediante as expectativas e desafios dos estudantes da graduação de enfermagem (ingressantes e concluintes) em uma instituição privada em município do estado de Rondônia. Métodos: Tratou-se de um de campo, com caráter exploratório-descritivo, de abordagem qualitativa. A amostragem foi composta por 20 acadêmicos, distribuídos da seguinte forma: 10 acadêmicos ingressantes e 10 concluintes. Resultados: A partir da observação empírica dos relatos, por meio dos dados convergentes comparamos as expectativas e desafios dos ingressantes aos concluintes e após exaustiva análise das falas dos participantes da pesquisa, emergiram três grupos temáticos, a saber: Motivações à Escolha da Graduação, Percepção Sobre o Papel do Enfermeiro e Expectativas e Desafios na Graduação. Conclusão: Pode-se notar a modificação dos discentes, nos seus pensamentos, forma de encarar desafios, trazendo novas expectativas mediante o futuro, servindo como um trampolim social, profissional e pessoal. Tal pesquisa poderá servir como demonstrativo dessa transformação, como a graduação modifica o discente e a maneira em que as expectativas são remodeladas e os desafios vencidos.
\end{abstract}

Palavras-chave: Enfermagem, Acadêmicos, Ensino superior.

\begin{abstract}
Objective: To understand the feelings experienced by academics regarding the expectations and challenges of undergraduate nursing students (freshmen and seniors) in a private institution in a city in the state of Rondônia. Methods: This was a field study, with an exploratory-descriptive character, with a qualitative approach. The sample consisted of 20 students, distributed as follows: 10 freshmen and 10 seniors. Results: From the empirical observation of the reports, through convergent data, we compared the expectations and challenges of freshmen to seniors, and after an exhaustive analysis of the research participants' statements, three thematic groups emerged, namely: Motivations for Choice of Graduation, Perception About the Nurse's Role and Expectations and Challenges in Graduation. Conclusion: It is possible to notice the modification of the students, in their thoughts, way of facing challenges, bringing new expectations towards the future, serving as a social, professional and personal springboard. Such research can serve as a demonstration of this transformation, how graduation changes the student and the way in which expectations are remodeled and challenges overcome.
\end{abstract}

Keywords: Nursing, Academics, University education.

\section{RESUMEN}

Objetivo: Comprender los sentimientos experimentados por los estudiantes con respecto a las expectativas y desafíos de los estudiantes de enfermería de pregrado (primer y último año) en una institución privada en una ciudad del estado de Rondônia. Métodos: Fue un estudio de campo, de carácter exploratorio-descriptivo, con abordaje cualitativo. La muestra estuvo conformada por 20 estudiantes, distribuidos de la siguiente manera: 10 estudiantes de primer año y 10 de último año. Resultados: A partir de la observación empírica

\footnotetext{
${ }^{1}$ Faculdade Interamericana de Porto Velho - UNIRON, Porto Velho - RO.

*E-mail: tainara.viana99@gmail.com
} 
de los informes, a través de datos convergentes, comparamos las expectativas y desafíos de los estudiantes de primer año con los de los de último año, y luego de un análisis exhaustivo de las declaraciones de los participantes de la investigación, surgieron tres grupos temáticos, a saber: Motivaciones para la elección de graduación, Percepción sobre el papel de la enfermera y sus expectativas y desafíos en la graduación. Conclusión: Es posible notar la modificación de los estudiantes, en sus pensamientos, forma de enfrentar los desafíos, trayendo nuevas expectativas hacia el futuro, sirviendo como trampolín social, profesional y personal. Tal investigación puede servir como demostración de esta transformación, cómo la graduación cambia al estudiante y la forma en que se remodelan las expectativas y se superan los desafíos.

Palabras clave: Enfermería, Académica, Enseñanza superior.

\section{INTRODUÇÃO}

Para a escolha da graduação em enfermagem, a influência do âmbito social em qual o indivíduo está inserido é significante. Da mesma maneira que no decorrer do curso este primeiro pensamento assume mudanças, justificado pelo contato com as disciplinas curriculares e o ensino prático vivenciado pelo acadêmico, podendo assumir uma mudança positiva ou negativa sobre a escolha da profissão (SILVA LAA, et al., 2019). Ao optar pela Enfermagem, o discente assume a escolha de cuidar de outras pessoas, podendo ser esse cuidado em seu nascimento ou até na morte, aprendendo assim a lidar melhor com suas limitações (MOURA IA, et al., 2016).

$\mathrm{Na}$ escolha de traçar o caminho da enfermagem, o estudante procura a realização social, esta visão tem a pretensão o cumprimento de expectativas e de projetos no âmbito pessoal, juntamente com a melhoria da condição social e autorrealização (GLERIANO JS, et al., 2017).O conhecimento das expectativas dos graduandos de enfermagem torna possível a análise e reflexão sobre os principais desafios para a formação acadêmica e as particularidades inerentes aos ingressantes na profissão da enfermagem (NASCIMENTO PN e BARBOSA MCL, 2017). De acordo com Neto ACA, et al. (2018), escolher um curso de nível superior é considerado uma tarefa difícil, cheia de ansiedades, dúvidas e incertezas e, também, de enormes responsabilidades, uma vez que as consequências dessa escolha podem resultar em insatisfação e desapontamento com o curso escolhido.

Ao discutir as expectativas e os desafios dos ingressantes e concluintes, elevam a significância da pesquisa, contribuindo para compreensão dos sentimentos vivenciados pelos acadêmicos durante este processo. Ofertando subsídios para melhorias na graduação com um olhar diferente para o discente, enxergando-o em sua totalidade. Buscou-se como objetivo, compreender os sentimentos vivenciados pelos acadêmicos mediante as expectativas e desafios dos estudantes da graduação de enfermagem (ingressantes e concluintes) em uma instituição privada no estado de Rondônia.

\section{MÉTODOS}

Tratou-se de um estudo de campo, com caráter exploratório-descritivo, de abordagem qualitativa. A pesquisa foi realizada na Instituição Faculdade Interamericana de um município localizado no estado de Rondônia, antecedendo a coleta de dados direta, foi indispensável buscar literaturas referente a graduação de enfermagem, dando enfoque para os ingressantes e concluintes. Os participantes da pesquisa, foram os acadêmicos de enfermagem matriculados no $1^{\circ} \mathrm{e} 10^{\circ}$ períodos, que aceitaram voluntariamente participar do estudo, maiores de 18 anos. Foram excluídos os participantes que se recusaram a assinar o TCLE, os que estavam cursando novamente alguma matéria incluída na grade do primeiro e décimo período.

A amostra foi de forma não probabilística, intencional ou proposital, constituída por 20 acadêmicos, a partir de um convite oral de colaboração realizado em particular por meio do WhatsApp aos ingressantes, e aos concluintes o convite oral. Os mesmos foram distribuídos da seguinte forma: 10 acadêmicos ingressantes e 10 concluintes. A coleta de dados foi realizada de forma adaptada devido a pandemia da COVID-19, por meio da aplicação de um questionário aberto, no qual foi utilizado um questionário para os ingressantes via GoogleForms e um para os concluintes impresso. Contendo cinco perguntas abertas para caracterização do perfil dos participantes e mais cincos perguntas referentes ao tema. 
Com as respostas obtidas as pesquisadoras agruparam em categorias, conforme as perguntas convergentes, mantendo na íntegra as respostas. Os dados coletados foram analisados com base no método de análise de conteúdo e comparados com os achados da literatura. Os participantes da pesquisa foram identificados pela sequência numérica de um a vinte, ficando assim distribuído do número 1 ao 10 os ingressantes, como: I1, 12, I3... e os concluintes do 11 ao 20, seguindo: $\mathrm{C} 11, \mathrm{C} 12$, C13.

Antes de realizar o estudo foi seguido os preceitos éticos e obtido a aprovação do CEP, com o número do parecer 4.381.517 e CAAE 38963120.9.0000.8028, aprovado no dia 05 de novembro de 2020 pela União Educacional do Norte (UNINORTE).

\section{RESULTADOS E DISCUSSÃO}

Mediante as questões de caracterização observamos os participantes pelo código de identificação e seus respectivos períodos que estão cursando. A prevalência do gênero feminino em ambos os períodos abordados. Referente a idade, na amostragem dos ingressantes há uma variável de 19 a 40 anos, e nos concluintes de 22 a 38 anos. Dentre a amostragem dos ingressantes, seis participantes se auto declararam pardo, dois declararam ser da raça branca e os outros dois da raça preta. Nos concluintes, seis participantes declararam ser da raça parda, e os outros quatros da raça branca (Quadro 1).

Quadro 1 - Caracterização dos participantes da pesquisa.

\begin{tabular}{|c|c|c|c|c|}
\hline $\begin{array}{c}\text { Código de } \\
\text { identificação }\end{array}$ & Idade & Gênero & Raça & $\begin{array}{c}\text { Período que está } \\
\text { cursando }\end{array}$ \\
\hline 11 & 19 & Feminino & Preta & $1^{\circ}$ \\
\hline I2 & 19 & Feminino & Preta & $1^{\circ}$ \\
\hline I3 & 20 & Feminino & Preta & $1^{\circ}$ \\
\hline 14 & 40 & Feminino & Parda & $1^{\circ}$ \\
\hline 15 & 21 & Feminino & Parda & $1^{\circ}$ \\
\hline 16 & 19 & Masculino & Pardo & $1^{\circ}$ \\
\hline 17 & 19 & Feminino & Branca & $1^{\circ}$ \\
\hline 18 & 20 & Feminino & Branca & $1^{\circ}$ \\
\hline 19 & 25 & Feminino & Parda & $10^{\circ}$ \\
\hline 110 & 19 & Masculino & Pardo & $10^{\circ}$ \\
\hline C11 & 22 & Feminino & Parda & $10^{\circ}$ \\
\hline C12 & 23 & Feminino & Parda & $10^{\circ}$ \\
\hline C13 & 36 & Feminino & Parda & $10^{\circ}$ \\
\hline C14 & 38 & Feminino & Parda & $10^{\circ}$ \\
\hline C15 & 32 & Feminino & Branca & $10^{\circ}$ \\
\hline C16 & 23 & Feminino & Parda & $10^{\circ}$ \\
\hline C17 & 31 & Feminino & Parda & $10^{\circ}$ \\
\hline C18 & 23 & Masculino & Branco & $10^{\circ}$ \\
\hline C19 & 23 & Feminino & Branca & \\
\hline C20 & 32 & Feminino & Branca & \\
\hline
\end{tabular}

Fonte: Viana TL, et al., 2021.

A partir da observação empírica dos relatos, por meio dos dados convergentes comparamos as expectativas e desafios dos ingressantes aos concluintes e após exaustiva análise das falas dos participantes da pesquisa, emergiram três grupos temáticos, a saber: Motivações à Escolha da Graduação, Percepção Sobre o Papel do Enfermeiro e Expectativas e Desafios na Graduação. 


\title{
Motivações à escolha da graduação
}

No discurso da escolha pela graduação em enfermagem os acadêmicos alegam a fala da vocação pela profissão, discurso este herdado por Florence Nightingale, a mulher que fundou a Enfermagem moderna e destacou-se no tratamento de feridos em guerras. Esta defendia a conciliação das técnicas científicas com o perfil servil, para uma assistência de enfermagem completa (SANEMATSU LSA, et al., 2019).

Em relação ao cuidado ao ser humano, a humanização se faz presente, orientada por condutas interdisciplinares, percepções e relações interpessoais, sendo que a enfermagem é parte mais atuante na abrangência do cuidado com o paciente sempre se baseando nas teorias de enfermagem existentes (SILVA NRF, et al., 2018). Foi demonstrado nas falas dos ingressantes e concluintes que a motivação para ingressar nesta graduação é o ato de prestar o cuidado e foi o que levou a maioria dos participantes da pesquisa a ingressar no curso de enfermagem.

\author{
"Sempre quis cuidar das pessoas" (17). \\ "Eu escolhi a enfermagem porque sempre gostei de prestar cuidados para as \\ pessoas [...]" (C12).
}

Para Gleriano JS, et al. (2017) compreender a motivação está conectado ao fato das peculiaridades da profissão, devido sua ligação ao tratar da humanidade. Ferreira MA, et al. (2020) afirma que a prática da enfermagem precisa ser baseada no conhecimento da ciência e tecnologias. Existindo um conceito superficial sobre esta profissão, entretanto ao ingressar no curso e no desenvolver dos períodos, é necessário que essa predefinição seja ampliada ao real conceito do que é a enfermagem, havendo uma evolução no pensamento sobre qual o papel do enfermeiro e quais as possíveis áreas de atuação, baseando a profissão na ciência.

Nos relatos dos concluintes ainda foi citado o interesse na escolha pertinente ao vasto campo de atuação, objetivando a atuação na área. O COFEN (2018), determina por meio da Resolução № 581/2018, uso de poderes conferidos pela lei $n^{\circ} 5.905$ de 12 de julho de 1973, nas grandes áreas de: Saúde Coletiva, Saúde da Criança e do Adolescente, Saúde do adulto (Saúde do homem e Saúde da mulher), Saúde do Idoso, Urgência e Emergência, Gestão, Ensino e Pesquisa. Vale ressaltar que tal texto corrobora com a fala do participante intitulado $\mathrm{C} 14$, eis a fala:

"Um dos motivos de escolher esse curso é o amplo campo de trabalho [...]" (C14).

Segundo Teodosio SSC e Padilha MI (2016), os ingressantes na graduação de enfermagem, carregam com eles alguns ideais sobre a profissão escolhida, sendo capaz de serem comprovados no desenvolvimento de sua formação, ou não. Um dos ideais é o amplo campo de atuação para o enfermeiro, torna-se um atrativo para a escolha da profissão, juntamente com a oferta de oportunidades devido à grande demanda de pessoas necessitadas da assistência de saúde.

\section{Percepção sobre do papel do enfermeiro}

Notou-se nas falas evidenciadas pelos ingressantes em relação ao papel do enfermeiro, intrinsicamente, o mito da subordinação entre os profissionais enfermeiro-médico, ou ainda limitados aos cuidados técnicos:

"Auxiliar o médico e demais colegas de trabalho no cuidado com os pacientes" (18).

"Prestar assistência ao paciente da melhor forma possível e controlar certas coisas como: medicamentos, alimentação e etc..." (11).

A enfermagem está entre as profissões com mais variedades em seu campo de atuação, apesar disso a mídia ainda circula os padrões históricos relacionados a esta profissão o que diminui o seu potencial, atraindo opiniões negativas (FERREIRA JCSCF, et al., 2020). Para desmistificar esse mito, se faz necessário a reprodução do conhecimento de que enfermagem é ciência, para combater os estereótipos criados culturalmente sobre a prática da profissão (JESUS MS e SAID FA, 2008). A consolidação do papel do enfermeiro é formada por meio de ensinamento dos docentes no decorrer da graduação. 
No período denominado "normal", fora da situação da pandemia da COVID-19, o trabalho do enfermeiro é considerado imprevisível, devido a intensidade da jornada dos trabalhadores e realização de multitarefas para atender às necessidades de gestão e de assistência com um ritmo de trabalho intrigante. Não remunerada a altura pelo trabalho de gestão, sendo minimizado à ideia de "enfermeiras auxiliando médicos nas condutas aos pacientes" (MELO CMM, et al., 2021). Visto que o impasse não é o discente ingressar com a percepção equivocada sobre o papel do enfermeiro, afinal este recebe uma definição rasa sobre o assunto. Mas sim um recém-formado enfermeiro, não compreender o seu papel de atuação, como consequência não exercer suas atividades de forma técnica-científica.

Para os concluintes, o papel que o enfermeiro exerce está mais consolidado, a assimilação desse papel citado, é de realizar uma assistência por meio do processo de enfermagem (PE) que está presente no cotidiano do enfermeiro em seu ambiente de trabalho, utilizando assim fatos baseado em ciência, para produzir uma assistência de cuidados.

\section{"É avaliar, planejar serviços da assistência de enfermagem" (15).}

"O enfermeiro tem como responsabilidade planejar, organizar e avaliar os serviços da assistência de enfermagem. Assim como prestar a assistência ao paciente de forma humanizada" (C11).

"Papel esse muito importante que vai desde planejar até oferecer uma assistência holística de boa qualidade a cada paciente/cliente [...]" (C15).

A assistência de enfermagem é planejada por meio da Sistematização da Assistência de Enfermagem (SAE) que organiza o serviço do profissional enfermeiro, com base teórica-cientifica, que possibilita a operacionalização do Processo de Enfermagem (PE) no qual é realizado anotações de enfermagem, auxiliando na organização e no planejamento da assistência prestada (OLIVEIRA MR, et al, 2019).

Ainda na área da saúde, os enfermeiros estão envolvidos na gestão, liderança além do cuidado direto aos pacientes, no qual muitas vezes tomam decisões de forma rápida e justa, assumem o papel de mediador de conflitos e respondem a situações de violência e vulnerabilidade (BECKER LG, et al., 2020). Sendo estes conceitos que os concluintes devem ter consigo, para exercer e expressar qual é o papel do enfermeiro.

\section{Expectativas e desafios na graduação}

Os ingressantes no ensino superior, são marcados pela transição da conclusão do nível médio para a inserção em uma nova fase, muita das vezes saindo da sua adolescência/juventude e migrando para a fase adulta. Entrando com desafios de adaptação e expectativas em relação ao curso escolhido, merecendo serem estimulados a buscarem alcançar suas expectativas (TORRES IL, et al., 2019). Para os ingressantes podemos perceber que as expectativas criadas são em relação ao aprendizado que irão ter durante todo o percurso da graduação.

\section{"Aprender" (17).}

"Aprender ao máximo tudo que me trata competência em minha profissão" (18).

Santos MS, et al. (2018) disserta sobre a aprendizagem ser a interação por meio do processo de ensino, na qual conhecimentos socialmente reconhecidos são extraídos. Tratando-se de aderir a uma rede complexa e dinâmica de interação entre professores e alunos para o desenvolvimento do conhecimento, sendo direcionado pelas metodologias escolhidas pelo docente. Vaccari A, et al. (2020) alegam que os alunos aprendem melhor quando praticam as habilidades em um ambiente apropriado, seguro e controlado; participando ativamente do seu processo de aprendizagem, devido a estimulação do aprendizado.

Para os concluintes as expectativas estão voltadas para a inserção no mercado de trabalho da sua área de formação. Os discentes do último período, estimulam a relação da assimilação do conhecimento obtido que promove os estudantes a dispor uma certa autonomia profissional, subordinado a aprendizagem científica, colaborando para um desempenho mais intelectivo e atitudinal (SANTOS El, et al., 2017). 
"Buscar um emprego [...]" (C18).

"[...] conseguir o primeiro trabalho na área da enfermagem [...]" (C19).

Da Cunha MA, et al. (2019), relatam que se inserir no meio profissional passa a ser um momento histórico na vida desse recém-formado sendo que, esse é um momento de deixar de ser acadêmico e lidar com o mundo fora da instituição. Desta forma, apesar dos sentimentos positivos em relação a conquistas e alcance de metas, o período universitário é crítico e causador de insegurança nos estudantes (LIMA BVBG, et al., 2017). Os últimos meses da graduação traz à tona um conjunto de emoções, de um lado a realização por estar concluindo o curso, por outro lado, existe a preocupação com o futuro incerto.

Em relação aos desafios, estamos imersos na sociedade que convive com o vírus da COVID-19, logo as aulas presenciais foram suspensas há mais de um ano. Isso consequentemente, mudou os desafios que os ingressantes enfrentam ao adentrar na graduação, nesse cenário específico. Para Bezerra IMP (2020), além do desafio geral da população de enfrentar uma pandemia do novo coronavírus, os cursos da saúde, entre eles o de enfermagem, tiveram que incorporar as tecnologias das aulas remotas, devido a necessidade da situação. Ainda com o cuidado de não se tornar apenas uma educação a distância. As falas dos ingressantes, expressam este desafio:

"[... a questão das aulas remotas, é um grande desafio atualmente para todos" (11).

"O desafio é assistir as aulas online, ainda somatizado ao contexto de pandemia [...]" (I10).

Bezerra IMP (2020), menciona o mundo virtual como um paradigma da educação, com seus benefícios e malefícios. Assim como Silveira A, et al. (2020) cita que além da dificuldade de obtenção e pelas restrições dos equipamentos técnicos, um grande desafio é a desmotivação de alunos e professores, como consequência da ausência de contato físico, tornando relacionamentos limitados. Sendo capaz de gerar dificuldade de interação entre professor e aluno, implicando na qualidade do processo de ensino. Somado as responsabilidades com os filhos, afazeres de casa, home office, entre outros.

Para os concluintes os desafios apresentados em comum aos ingressantes foram, os associados a realidade da pandemia. Não apenas com as aulas remotas, como também atuar na linha de frente por meio do estágio supervisionado:

\section{"[...] vivenciar o campo de estágio em um momento tão difícil” (C13).}

"O desafio nesta reta final é de que será que vou querer exercer a profissão no momento tão difícil em que estamos vivendo [...]" (C14).

"O desafio está sendo enfrentar uma pandemia mundial e as aulas remotas" (C18).

Durante o combate a pandemia da COVID-19 a enfermagem exerce uma função relevante, desempenhando a assistência aos pacientes durante um período prolongado, tornando essa classe mais suscetível ao vírus. Recebendo ainda o apoio dos estudantes de enfermagem que estão em seu último ano de graduação (SOUZA LB, et al., 2020).

Atuar durante o estágio supervisionado em uma pandemia traz riscos e inseguranças devido à grande facilidade de contaminação, entretanto participar da linha de frente durante esse momento único e histórico vivido nos tempos atuais é um momento ímpar na vida dos concluintes, podendo ser um diferencial em suas carreiras por trazer um conhecimento singular. Franzoi MAH e Cauduro FLF (2020), levanta está questão sobre estudantes atuarem na linha de frente, sendo sujeitos a possível contaminação e por isso desenvolverem sentimentos de ansiedade e vulnerabilidade. Os autores ainda complementam que somado a isto, existe a chance rara de aprender e contribuir, agregado a gratificação pessoal em fazer parte da história.

Um outro desafio levantado é a falta do tempo, que é a realidade de muitos estudantes da graduação de enfermagem em uma instituição privada, que geralmente optam pelo período noturno por passarem o dia trabalhando. Alguns em trabalhos diversos, e outros atuam como técnico de enfermagem, alterando sua escala para conciliar com as aulas. 
"O tempo, infelizmente trabalhar e estudar é muito difícil. Às vezes você está atolado de trabalho e atividade pra fazer e acaba causando uma exaustão mental [...]"(I3).

Santos JER, et al. (2020) relatam que existem inúmeras as adversidades de se trabalhar e estudar simultaneamente, como o estresse, a sonolência, o cansaço mental, a falta de tempo para se relacionar com os familiares e ausência de tempo para se dedicar aos estudos, entre diversas outras dificuldades, sendo assim é necessário muita determinação para que o estudante não perca a motivação e o foco em alcançar a meta que é a conclusão do ensino superior e seus benefícios prometidos: ter uma boa renda salarial, adquirir novos conhecimentos, possuir maior qualificação e espaço no mercado de trabalho.

Foi relatado a sobrecarga, referente ao último semestre, no qual é realizado o Trabalho de Conclusão de Curso, o estágio supervisionado, além de outras tarefas na área pessoal. Ainda temos os casos dos concluintes que tem sua jornada de trabalho, tornando este último semestre ainda mais pesado.

"Meu grande desafio é manter o convívio familiar, sair com amigos e ao mesmo tempo cumprir as obrigações acadêmicas, que costumam causar situações estressantes no dia a dia [...]" (C12).

"Acordar cedo, o estágio supervisionado, trabalhar no outro horário, coletar os dados para o TCC[...]" (C16).

A vida acadêmica sujeita os estudantes a múltiplos fatores estressantes, podendo afetar a sua qualidade de vida, ao demandar gerenciamento da vida pessoal e social, juntamente com os deveres acadêmicos e os preparativos para a carreira profissional. Podendo causar sentimentos como angústia, medo, desgaste físico, pois além da carga de estágios necessários, existe ainda a tarefa de desenvolver o trabalho de conclusão de curso (MUSSI FC, et al., 2019).

Freitas AMC, et al. (2017) cita que com o suceder dos semestres é promovido um aumento progressivo do estresse, concebido pelas atividades da vida universitária, como o trabalho de conclusão de curso e estágio supervisionado para os últimos períodos. Com uma intensificação das obrigações e atividades, como metodologia para fornecer embasamento técnico científico aos graduandos, podendo afetar a sua qualidade de vida.

Outrossim, o sentimento relatado pelos concluintes, foi a insegurança em relação a própria capacidade como enfermeiro referente a prática e a sua inserção no mercado de trabalho.

"A questão da insegurança, preciso acreditar mais que sou capaz, afinal não é à toa que cheguei até aqui basta apenas eu acreditar mais nisso" (C11).

"[...] Outras questões são os medos de logo após concluir não entrar no mercado de trabalho devido à falta de prática, o sentimento de insegurança" (C15).

A passagem da graduação para a vida profissional está relacionada a várias tomadas de decisões, todas elas de cunho relevante, pois modificará o estilo de vida do novo enfermeiro. Aquele que antes estava em uma zona de conforto durante anos, que era o período acadêmico, começa a encarar importantes escolhas. Tais como a forma de criar sua independência, responsabilidades financeiras, inserção no mercado de trabalho e afins. Provocando sensação de desconforto para os concluintes (FREITAS ACM, et al., 2018).

\section{CONCLUSÃO}

Pode-se notar a modificação dos discentes, nos seus pensamentos, forma de encarar desafios, trazendo novas expectativas mediante o futuro, servindo como um trampolim social, profissional e pessoal. Ao adentrar os ingressantes engrenam com a sua bagagem social, trazendo consigo seus ideais para a graduação. Por sua vez os concluintes, levam o aprendizado dos anos que passaram como base para a carreira na enfermagem. Tal pesquisa poderá servir como demonstrativo dessa transformação, como a graduação modifica o discente e a maneira em que as expectativas são remodeladas e os desafios vencidos. 


\section{REFERÊNCIAS}

1. BECKER LG, et al. Conhecimento dos enfermeiros sobre o novo código de ética do profissional de enfermagem. Revista Nursing. 2020; 23(271): 5041-5047.

2. BEZERRA, IMP. Estado da arte sobre o ensino de enfermagem e os desafios do uso de tecnologias remotas em época de pandemia da corona vírus. J Hum Growth. Dez. 2020; 30(1):141-147.

3. COFEN, Conselho Federal da enfermagem. Resolução COFEN, n581/2018.

4. CUNHA MA, et al. A inserção profissional de enfermeiros recém-formados. Revista GepesVida, 2019; 5(10).

5. FERREIRA JCSC, et al. Onde está a enfermagem? A (in)visibilidade desta categoria profissional nos meios de comunicação. Enferm. Foco 2020; 11(2): 50-56.

6. FERREIRA MA, et al. Fundamentos Nightingaleanos, cuidado humano e políticas de saúde no Século XXI. Rev enferm UERJ, Rio de Janeiro, 2020; 28: e50353.

7. FRANZOI MAH, CAUDURO FLF. Atuação de estudantes de enfermagem na pandemia de covid-19. Cogitare Enfermagem, 2020; 5(25).

8. FREITAS AMC, et al. Percepções de estudantes de enfermagem referente à qualidade de vida na trajetória acadêmica. Rev Enferm UFSM 2017; 7(2).

9. GLERIANO JS, et al. Perfil e significados para a formação em enfermagem. Revista Saúde e Desenvolvimento, 2017; $11(6)$.

10. JESUS MS, SAID FA. Autonomia e a prática assistencial do enfermeiro. Cogitare Enfermagem, 2008; 13(3).

11. LIMA BVBG, et al. Avaliação da ansiedade e autoestima em concluintes do curso de graduação em enfermagem. Rev enferm UFPE on line, Recife, nov, 2017.

12. MELO CMM, et al. Pandemia da covid-19: algo de novo no trabalho da enfermeira?. Rev. Baiana Enferm., Salvador, $2021 ; 35$.

13. MOURA IH, et al. Qualidade de vida de estudantes de graduação em enfermagem. Rev Gaúcha Enferm. 2016; 37(2).

14. MUSSI FC, et al. Comparação do estresse em universitários de enfermagem ingressantes e concluintes do curso. Revista da Escola de Enfermagem da USP, 2019; 53.

15. NASCIMENTO PN, BARBOSA MCL. Perspectivas dos graduandos de enfermagem frente ao mercado de trabalho. Id on Line Rev. Psic. 2017; 11(35).

16. NETO ACA, et al. Uma proposta de análise da evasão no ensino superior do ifba. Ensino em Foco, $2018 ; 1$ (1).

17. OLIVEIRA MR, et al. Sistematização da assistência de enfermagem: percepção e conhecimento da enfermagem Brasileira. Revista Brasileira de Enfermagem, 2019; 72(6).

18. SANEMATSU LSA, et al. A produção científica sobre vocação na enfermagem. Rev enferm UFPE on line., Recife, $2019 ; 13(3)$.

19. SANTOS El, et al. Comparative study of representations of professional autonomy produced by first and last-period undergraduate nursing students. Rev. Latino-Am. Enfermagem. 2017; 25: e2927.

20. SANTOS JER, et al. Estudar e trabalhar: motivações e dificuldades de graduandos de Enfermagem. Nursing [Internet], $2020 ; 23(262)$.

21. SANTOS MS, et al. Acolher mais: recepção dos calouros do curso de graduação em enfermagem. Revista graduação/pós graduação em educação, 2018.

22. SILVA LAA, et al. Arquétipos discentes: percepções de docentes de enfermagem. Rev Fun Care Online. 2019 jul/set; 11(4): 1081-1087.

23. SILVA NRF, et al. Teorias de enfermagem aplicadas no cuidado a pacientes oncológicos: contribuição para prática clínica do enfermeiro. Revista Uningá, 2018; 55(2).

24. SILVEIRA A, et al. Estratégias e desafios do ensino remoto na Enfermagem. Enfermagem em Foco, 2021; 11(5).

25. SOUZA LB, et al. Estágio curricular supervisionado em enfermagem durante a pandemia de Coronavírus: experiências na atenção básica. J. nurs. health. 2020; 10(n.esp.): e20104017.

26. TEODOSIO SSC, PADILHA MI. "Ser enfermeiro": escolha profissional e a construção dos processos identitários (anos 1970). Rev Bras Enferm 2016; 69(3): 401-7.

27. TORRES IL, et al. Recepção de ingressantes universitários: experiência construtivista relacional de discentes de enfermagem para integração. REFACS (online) 2019; 7(1): 91-96.

28. VACCARI A, et al. Implementação de um modelo para roteiros de aula no laboratório de enfermagem: fortalecendo a aprendizagem. Rev. Gaúcha Enferm., Porto Alegre, 2020; 41. 